\title{
Towards an asymptotically safe completion of the Standard Model*
}

\author{
Kamila Kowalska ${ }^{\dagger}$ \\ Technische Universität Dortmund \\ National Centre for Nuclear Research, Warsaw \\ E-mail: kamila.kowalska@ncbj.gov.pl
}

Andrew Bond

University of Sussex

E-mail: a.bondesussex.ac.uk

Gudrun Hiller

Technische Universität Dortmund

E-mail: gudrun.hillereuni-dortmund.de

\section{Daniel Litim}

University of Sussex

E-mail: d.litimesussex.ac.uk

\begin{abstract}
We explore the possibility to UV-complete the Standard Model in an asymptotically safe manner. We assume the existence of $N_{F}$ new types of vector-like fermions which minimally couple to $S U(3)_{C}$ and/or $S U(2)_{L}$ and modify the running of the corresponding gauge couplings in such a way that at least one of them is not asymptotically free anymore. If additionally the beyond the Standard Model fermions carry a non-zero hypercharge, the $U(1)_{Y}$ gauge coupling can become asymptotically free, thus avoiding the problem of the Landau Pole. We classify the emerging UV fixed points and discuss their dependence on transformation properties of the new fermions under the gauge symmetries. We also show that additional constraints on the structure of the beyond the Standard Model sector arise if one requires an asymptotically safe UV fixed point to be connected to the Standard Model through a well-defined renormalization group trajectory.
\end{abstract}

The European Physical Society Conference on High Energy Physics

5-12 July

Venice, Italy

\footnotetext{
*DO-TH 17/23, QFET-2017-16
}

${ }^{\dagger}$ Speaker. 


\section{Introduction}

Asymptotic safety states that fundamental quantum fields may remain interacting (rather than a free) at highest energies [1,2], implying that running couplings reach an interacting ultraviolet (UV) fixed point under the renormalization group evolution. Asymptotic safety has initially been proposed as a scenario for quantum gravity [2]. More recently, necessary and sufficient conditions for asymptotic safety in general 4-dimensional weakly coupled gauge theories have been derived [3,4]. Most importantly, it was found that Yukawa interactions together with elementary scalar fields such as the Higgs are crucial to tame the high-energy behavior of the running couplings. The feasibility of asymptotic safety is thus well motivated theoretically and opens intriguing new directions for model building beyond the Standard Model (BSM).

In these proceedings we report on the results presented in details in [5]. Our goal is to understand whether and how minimal extensions of the SM can be found with weakly interacting UV fixed points. Our central new input are BSM fermions and scalars, some of which are charged under the gauge symmetries of the SM. We are particularly interested in the concrete conditions under which interacting UV fixed points are connected through well-defined trajectories with the SM at low energies.

\section{Basics of asymptotic safety}

To illustrate the mechanism of asymptotic safety, we consider the renormalization group (RG) flow for a simple gauge theory with a gauge coupling $\alpha_{g}=g^{2} /(4 \pi)^{2}$. The theory encompasses also scalars and fermions with Yukawa interactions described by a coupling $\alpha_{y}=y^{2} /(4 \pi)^{2}$. Within perturbation theory, the RG flow in the gauge-Yukawa system is given, to the leading non-trivial order, by

$$
\begin{aligned}
& \beta_{g} \equiv \frac{d \alpha_{g}}{d \ln \mu}=\left(-B+C \alpha_{g}-D \alpha_{y}\right) \alpha_{g}^{2}, \\
& \beta_{y} \equiv \frac{d \alpha_{y}}{d \ln \mu}=\left(E \alpha_{y}-F \alpha_{g}\right) \alpha_{y} .
\end{aligned}
$$

The gauge coupling is asymptotically free (infrared free) provided that the one loop gauge coefficient obeys $B>0(B<0)$. The two loop gauge coefficient $C$ may take either sign depending on the matter content. The other loop coefficients obey $D, E, F>0$ for any quantum field theory.

In general, theories with Eq. (2.1) may have various types of fixed points, depending on the matter content. Equating $\beta_{i}=0$ for both couplings, three types of fixed points are found. The Gaussian fixed point $\left(\alpha_{g}^{*}, \alpha_{y}^{*}\right)=(0,0)$ always exists, and corresponds to the UV (IR) fixed point provided that $B>0(B<0)$. An interacting fixed point where Yukawa interactions are switchedoff may also exist, with $\left(\alpha_{g}^{*}, \alpha_{y}^{*}\right)=\left(\frac{B}{C}, 0\right)$. This is the well-known Caswell-Banks-Zaks fixed point $[6,7]$ which requires $B \cdot C>0$ to be physical and $B / C \ll 1$ to be perturbative. Finally, a fully interacting gauge-Yukawa fixed point may arise provided that the Yukawa coupling is nonvanishing, $\left(\alpha_{g}^{*}, \alpha_{y}^{*}\right)=\left(\frac{B}{C^{\prime}}, \frac{B}{C^{\prime}} \frac{F}{E}\right)$, where $C^{\prime}=C-D \frac{F}{E}$. For theories without asymptotic freedom $(B<0)$ the gauge coupling may now take a viable interacting fixed point $\alpha_{g}^{*}=B / C^{\prime}>0$ as long as $C^{\prime}<0$. This is the interacting UV fixed point of asymptotic safety. 


\section{Asymptotically safe extensions of the Standard Model}

Asymptotic safety in the extensions of the SM requires the presence of new matter fields which carry charges under the SM gauge groups and thereby modify the RG running of the couplings. Guided by the findings of [3, 4], we consider the existence of $N_{F}$ flavors of BSM vector-like fermions $\psi$ which minimally couple to the SM gauge bosons. In general, the BSM fermions may carry charges under $S U(3)_{C}, S U(2)_{L}$, or hypercharge $Y$, meaning

$$
\psi_{i}\left(R_{3}, R_{2}, Y\right)
$$

where $i=1, \cdots, N_{F}$ denotes the flavor index. They couple via Yukawa interactions to complex scalar fields $S_{i j}$ which we assume to be singlets under the SM. Since the BSM fermions are taken to be vector-like, gauge anomalies are not an issue. The Yukawa interactions are given by

$$
L_{\mathrm{BSM}, \text { Yukawa }}=-y \operatorname{Tr}\left(\bar{\psi}_{\mathrm{L}} \mathrm{S} \psi_{\mathrm{R}}+\bar{\psi}_{\mathrm{R}} \mathrm{S}^{\dagger} \psi_{\mathrm{L}}\right) .
$$

Here, $y$ denotes the BSM Yukawa coupling, the trace Tr sums over color and flavor indices, and the decomposition $\psi=\psi_{L}+\psi_{R}$ with $\psi_{R / L}=\frac{1}{2}\left(1 \pm \gamma_{5}\right) \psi$ is understood. We neglect at this point the role of quartic self interactions of the BSM scalars, as well as their portal couplings to the Higgs boson. The reason is that, at weak coupling, neither of these are relevant for the primary existence of the UV fixed point in the gauge-Yukawa sector.

Firstly, we limit ourselves to BSM fermions which carry no hypercharge. Consequently, the free fundamental parameters of the BSM matter sector are given by their group-theoretical representation under $S U(2)_{L}$ and $S U(3)_{C}$, and their flavor multiplicity $N_{F}$. The corresponding RG equations to the leading non-trivial order are given by $[8,9,10]$

$$
\begin{aligned}
& \beta_{3} \equiv \frac{d \alpha_{3}}{d \ln \mu}=\left(-B_{3}+C_{3} \alpha_{3}+G_{3} \alpha_{2}-D_{3} \alpha_{y}\right) \alpha_{3}^{2}, \\
& \beta_{2} \equiv \frac{d \alpha_{2}}{d \ln \mu}=\left(-B_{2}+C_{2} \alpha_{2}+G_{2} \alpha_{3}-D_{2} \alpha_{y}\right) \alpha_{2}^{2}, \\
& \beta_{y} \equiv \frac{d \alpha_{y}}{d \ln \mu}=\left(E \alpha_{y}-F_{2} \alpha_{2}-F_{3} \alpha_{3}\right) \alpha_{y},
\end{aligned}
$$

where the loop coefficients depend on $R_{2}, R_{3}$ and $N_{F}$. Gauge-Yukawa theories with Eq. (3.3) may display up to four different types of weakly coupled UV fixed points, depending on whether the gauge couplings take free or interacting values in the UV. They are summarized in Table 1.

The existence of a UV fixed point depends on the number of the BSM fermions $N_{F}$ and their transformation properties under $S U(3)_{C} \otimes S U(2)_{L}$. For example, for a partially interacting fixed point $\mathbf{F P}_{\mathbf{3}}$, a minimal requirement to make it a UV fixed point is the loss of asymptotic freedom in the $S U(3)_{C}$ gauge sector, $B_{3}<0$, translating into a lower bound on the number of the BSM fermions, $N_{F}>21 /\left(4 S_{2}\left(R_{3}\right) d\left(R_{2}\right)\right)$. Furthermore, the physicality condition of the couplings at the fixed point translates into a condition $D_{3} F_{3}-E C_{3}>0$. In Table 2 we show the maximal number of BSM vector-like fermions compatible with asymptotic freedom, $N_{\mathrm{AF}}$, for $S U(2)_{L}$ singlets, doublets and triplets, and for different dimensions of the $S U(3)_{C}$ representations. Asymptotic freedom is lost as soon as the BSM fermions transform under higher-dimensional representations of the gauge group. We also show the smallest number of flavors required for an asymptotically safe fixed 


\begin{tabular}{c|cc|c|c|c}
\hline case & \multicolumn{2}{|c|}{ gauge couplings } & BSM Yuk & type & info \\
\hline $\mathbf{F P}_{\mathbf{1}}$ & $\alpha_{3}^{*}=0$ & $\alpha_{2}^{*}=0$ & $\alpha_{y}^{*}=0$ & $\mathbf{G} \cdot \mathbf{G}$ & non-interacting \\
$\mathbf{F P}_{\mathbf{2}}$ & $\alpha_{3}^{*}=0$ & $\alpha_{2}^{*}>0$ & $\alpha_{y}^{*}>0$ & $\mathbf{G} \cdot \mathbf{G Y}$ & partially interacting \\
$\mathbf{F P}_{\mathbf{3}}$ & $\alpha_{3}^{*}>0$ & $\alpha_{2}^{*}=0$ & $\alpha_{y}^{*}>0$ & $\mathbf{G Y} \cdot \mathbf{G}$ & partially interacting \\
$\mathbf{F P}_{\mathbf{4}}$ & $\alpha_{3}^{*}>0$ & $\alpha_{2}^{*}>0$ & $\alpha_{y}^{*}>0$ & $\mathbf{G Y} \cdot \mathbf{G Y}$ & fully interacting \\
\hline
\end{tabular}

Table 1: Four different types of UV fixed points $\mathbf{F P}_{\mathbf{1}}-\mathbf{F P}_{\mathbf{4}}$ in minimal BSM extensions of the SM with 3.3. We also indicate how the fixed points can be interpreted as products of the Gaussian $(\mathrm{G})$ and gauge-Yukawa (GY) fixed points when viewed from the individual gauge group factors.

point $\mathbf{F P}_{\mathbf{3}}$ to exists, $N_{\mathrm{AS}}$. For fermions in the fundamental representations, no asymptotically safe solution is possible. For fermions in higher-dimensional representations, we find that a fixed point exists for sufficiently large $N_{F}$. The same is true for $\mathbf{F P}_{2}$, with interchange of the indices $2 \rightarrow 3$ and $3 \rightarrow 2$. For a fully interacting fixed point $\mathbf{F P}_{4}$ to exists, the BSM fermions must transform in a representation higher than fundamental with respect to at least one of the symmetry groups.

In the vicinity of interacting fixed points, the running of couplings is power law like, characterized by universal scaling exponents $\left\{\vartheta_{i}\right\}$. Linearizing the RG flow in the vicinity of a fixed point, $\beta_{i}=\sum_{j} M_{i j}\left(\alpha_{j}-\alpha_{j}^{*}\right)+$ subleading, the scaling exponents can be derived as the eigenvalues of the stability matrix $M_{i j}=\partial \beta_{i} /\left.\partial \alpha_{j}\right|_{*}$. Eigendirections are termed relevant (irrelevant) provided that $\vartheta<0(\vartheta>0)$. Marginal couplings have vanishing eigenvalues at linear order. Any RG trajectory emanating from a UV fixed point qualifies as a UV complete quantum field theory. The set of such UV-safe trajectories is called a critical surface and its dimensionality is determined by the relevant and marginally relevant couplings in the UV. Conversely, the irrelevant couplings are uniquely fixed in the UV by the relevant couplings. Consequently, the number of fundamentally free parameters which characterize the UV-safe trajectories is given by the dimensionality of the critical surface. At low energies, physically viable BSM trajectories must connect with those of the $\mathrm{SM}$, as soon as the BSM matter fields have decoupled.

At partially interacting fixed points, one of the two gauge couplings remains asymptotically free, while the other one becomes asymptotically safe. Moreover, the asymptotically (free) safe coupling is (marginally) relevant and the UV critical surface is invariably two-dimensional. On the

\begin{tabular}{ccccccc}
\hline & \multicolumn{2}{c}{$\mathbf{R}_{\mathbf{2}}=\mathbf{1}$} & \multicolumn{2}{c}{$\mathbf{R}_{\mathbf{2}}=\mathbf{2}$} & \multicolumn{2}{c}{$\mathbf{R}_{\mathbf{2}}=\mathbf{3}$} \\
$\mathbf{R}_{\mathbf{3}}$ & $N_{\mathrm{AF}}$ & $N_{\mathrm{AS}}$ & $N_{\mathrm{AF}}$ & $N_{\mathrm{AS}}$ & $N_{\mathrm{AF}}$ & $N_{\mathrm{AS}}$ \\
\hline $\mathbf{3}$ & 10 & - & 6 & - & 3 & - \\
$\mathbf{6}$ & 2 & 37 & 1 & 77 & - & 116 \\
$\mathbf{8}$ & 1 & 95 & - & 198 & - & 299 \\
$\mathbf{1 0}$ & - & 17 & - & 34 & - & 51 \\
$\mathbf{1 5}$ & - & 30 & - & 60 & - & 90 \\
$\mathbf{1 5}^{\prime}$ & - & 17 & - & 33 & - & 50 \\
\hline
\end{tabular}

Table 2: Asymptotic freedom versus asymptotic safety at the partially interacting fixed point $\mathbf{F P}_{\mathbf{3}}$. Shown are the maximal numbers of BSM fermion flavors compatible with asymptotic freedom, $N_{\mathrm{AF}}$, and the smallest number of flavors required for an asymptotically safe fixed point $\mathbf{F P}_{3}$ to exist, $N_{\mathrm{AS}}$, both in dependence on the fermion representations $R_{2}$ and $R_{3}$ under $S U(2)_{L}$ and $S U(3)_{C}$, respectively. Perturbative couplings are assumed, $0<\alpha_{3}^{*}, \alpha_{y}^{*}<1$. 


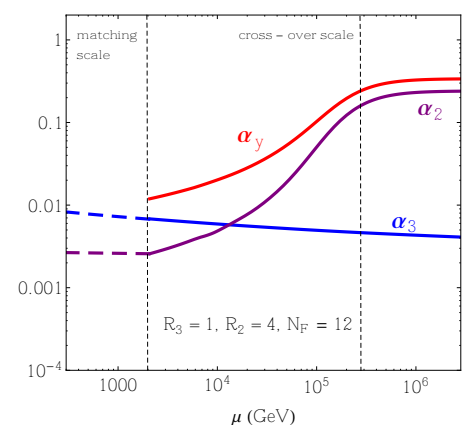

(a) Model A $\left(\mathbf{F P}_{2}\right)$

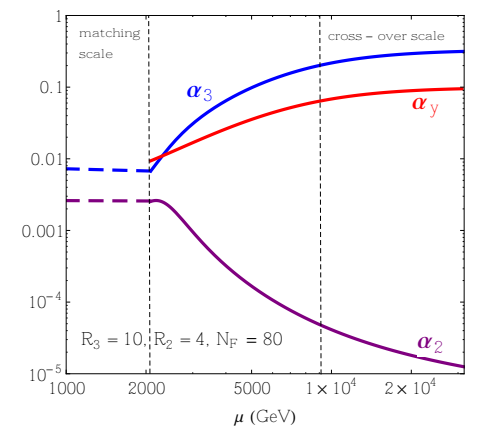

(b) Model C ( $\left.\mathbf{F P}_{\mathbf{3}}\right)$

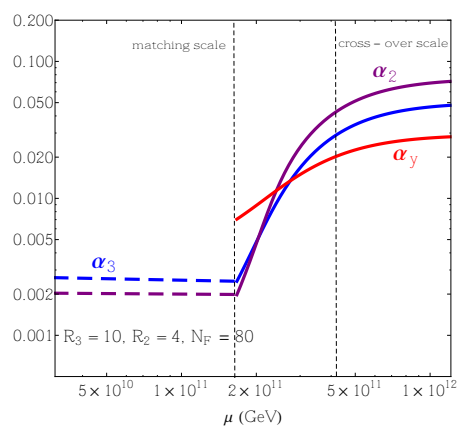

(c) Model C $\left(\mathbf{F P}_{4}\right)$

Figure 1: Matching of the UV interacting fixed points onto the SM. BSM (SM) running is shown by full (dashed) lines. The matching and the cross-over scales are indicated as vertical dotted lines.

other hand, the BSM Yukawa coupling, $\alpha_{y}$, is irrelevant and fully specified by the asymptotically safe coupling in the UV. A convenient choice for the two fundamentally free dimensionless parameters which characterize UV-safe trajectories running out of the fixed point are the deviations of the gauge couplings from their UV fixed point values at some high scale. In the immediate vicinity of the UV fixed point the RG flow is of the power-law type and thus fast, controlled by the relevant scaling exponent. Further away from the fixed point, as soon as $\alpha_{\mathrm{AS}} \approx 2 / 3 \alpha_{\mathrm{AS}}^{*}$ and below [11], we observe a cross-over whereby the running becomes logarithmically slow instead, dominated by the "would-be" Gaussian IR fixed point. Two examples of such a behavior are shown in Fig.1a and Fig.1b for the fixed points $\mathbf{F P}_{\mathbf{2}}$ and $\mathbf{F P}_{3}$, respectively. Note that the matching scale is a free parameter and can be chosen arbitrarily.

All fully interacting UV fixed points $\mathbf{F P}_{\mathbf{4}}$ are characterized by a stability matrix with a single relevant eigenvalue. This result has important implications. Unlike in asymptotically free theories (or in asymptotically safe theories with partially interacting fixed points) where every gauge coupling corresponds to a UV relevant direction, here, instead, the UV critical surface is of a lower dimensionality. As a consequence, the number of fundamentally independent parameters is reduced, leading to an enhanced level of predictivity. In our models, the UV critical surface at fully interacting UV fixed points becomes one-dimensional, parametrized by a single free parameter. Consequently, only one out of the three couplings $\left(\alpha_{3}, \alpha_{2}, \alpha_{y}\right)$ may be considered as an independent variable. Since the set of determining equations is over-constrained, a successful matching cannot be guaranteed from the outset, meaning that the viability needs to be checked for each case. In Fig.1c we present an example, in which the matching is possible at the high-energy scale.

Finally, we will shorty comment on a scenario in which the BSM fermions carry a non-zero hypercharge [12]. In this case, two qualitatively different asymptotic types of behavior of the $U(1)_{Y}$ gauge coupling can be observed. In any of the models considered above, there is always a lower bound on the value of the hypercharge, above which $\alpha_{1}$ becomes asymptotically free. This is illustrated in Fig.2a for a scenario with the fixed point $\mathbf{F P}_{2}$. Alternatively, one may assume that the BSM fermions are singlets under the $S U(3)_{C} \otimes S U(2)_{L}$ gauge symmetry. In such a case, a minimal value of hypercharge exists for which $\alpha_{1}$ becomes asymptotically safe. The RG running towards this new type of a partially interacting UV fixed point, $\mathbf{F P}_{\mathbf{Y}}$, is shown in Fig.2b. 


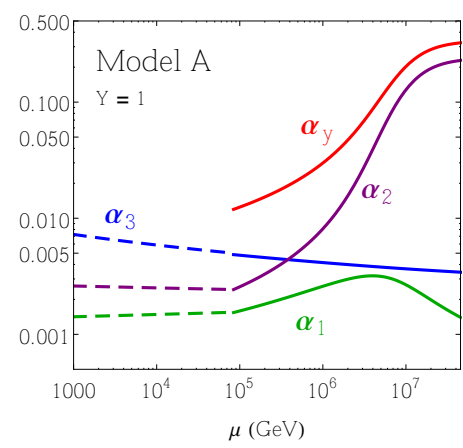

(a) Model A $\left(\mathbf{F P}_{2}\right)$

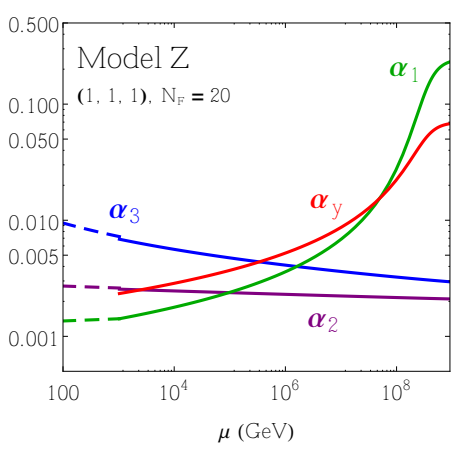

(b) Model Z (FPY)

Figure 2: Matching of the UV fixed points onto the SM. BSM (SM) running is shown by full (dashed) lines.

\section{Conclusions}

We investigated asymptotically safe extensions of the SM, constructed by adding new fermions and scalar singlet fields. The new matter fields interact via a single Yukawa coupling to help generate interacting UV fixed points. A large variety of stable high energy fixed points emerges where either the strong, the weak, the hypercharge, or any two of the gauge couplings assume finite values. Many of the fully interacting fixed points can also be matched onto the SM including at $\mathrm{TeV}$ scales, leading to signatures that could be observed at the colliders. An intriguing feature of models with fully interacting UV fixed points is a relation between gauge couplings, dictated by asymptotic safety. The number of fundamentally free parameters is thereby reduced offering an enhanced degree of predictivity compared to the SM, quite similar to the idea of unification.

\section{References}

[1] K. G. Wilson, Phys. Rev. B 4, 3174 (1971).

[2] S. Weinberg, "Ultraviolet Divergences In Quantum Theories Of Gravitation".

[3] D. F. Litim and F. Sannino, JHEP 1412, 178 (2014) [arXiv:1406.2337 [hep-th]].

[4] A. D. Bond and D. F. Litim, Eur. Phys. J. C 77, no. 6, 429 (2017) Erratum: [Eur. Phys. J. C 77, no. 8, 525 (2017)] [arXiv: 1608.00519 [hep-th]].

[5] A. D. Bond, G. Hiller, K. Kowalska and D. F. Litim, JHEP 1708, 004 (2017) [arXiv:1702.01727 [hep-ph]].

[6] W. E. Caswell, Phys. Rev. Lett. 33, 244 (1974).

[7] T. Banks and A. Zaks, Nucl. Phys. B 196, 189 (1982).

[8] M. E. Machacek and M. T. Vaughn, Nucl. Phys. B 222, 83 (1983).

[9] M. E. Machacek and M. T. Vaughn, Nucl. Phys. B 236, 221 (1984).

[10] M. E. Machacek and M. T. Vaughn, Nucl. Phys. B 249, 70 (1985).

[11] D. F. Litim, M. Mojaza and F. Sannino, JHEP 1601, 081 (2016) [arXiv:1501.03061 [hep-th]].

[12] A. D. Bond, G. Hiller, K. Kowalska and D. F. Litim, to appear. 